\title{
EVALUACIÓN DE ALTERNATIVAS BIOLÓGICAS PARA EL CONTROL DE ASCOCHYTA SPP. EN EL CULTIVO DE ARVEJA, PAMPLONA, NORTE DE SANTANDER
}

\author{
EVALUATION OF BIOLOGICAL ALTERNATIVES FOR THE CONTROL OF \\ ASCOCHYTA SPP. IN THE PEA CROP, PAMPLONA, NORTE DE SANT ANDER
}

\author{
Joe Manuel Torrado Martínez ${ }^{1}$, Leónides Castellanos González ${ }^{2}$, \\ Nestor Céspedes Novoa ${ }^{3}$ \\ Entidad \\ ${ }^{1}$ Ingeniero Agrónomo. Universidad de Pamplona, Colombia. \\ joe.torrado@hotmail.com \\ ${ }^{2}$ PhD. En Ciencias Agrícolas. Universidad de Pamplona. Colombia. \\ Iclcastell@gmail.com \\ ${ }^{3}$ Administrador Ambiental y de los Recursos Naturales. ASPAGRO, \\ Pamplona, Colombianestorcespedeshse@gmail.com
}

\section{Resumen}

El tizón por el complejo Ascochyta es una seria enfermedad de la alverja en Pamplona, Norte de Santander la cual requiere del uso frecuente de fungicidas químicos a pesar de que han sido informada la posibilidad del uso de la lucha biológica. El objetivo de la presente investigación fue evaluar la eficacia de alternativas biológicas para el control de Ascochyta spp. en el cultivo de arveja (Pisum sativum) bajo las condiciones de Pamplona. Se condujo una investigación experimental, donde se evaluaron seis tratamientos: tres con biopreparados producidos por la Asociación de Productores Agropecuarios (ASPAGRO), solos o combinados, un antagonista comercial (Trichoderma harzianum), un tratamiento químico con benomil, y un testigo. Se determinó la incidencia de la enfermedad, el Área bajo la Curva de Progreso de la Enfermedad (ABCPE). Además, se compararon en los tratamientos el número de vainas por plantas, el numero de granos por vaina, el peso de 10 granos y el rendimiento. El biopreparado Caldo Rizósfera de ASPAGRO y el biopreparado comercial a base de Trichoderma logran reducir la incidencia y el ABCPE del complejo Ascochyta spp. en arveja de forma similar al fungicida químico benomil, obteniéndose los niveles de rendimiento del cultivo similares a los del tratamiento químico.

Palabras clave

Pisum sativum, Trichoderma, microrganismos eficientes, medio ambiente 


\begin{abstract}
The blight caused by Ascochyta complex is a serious pea disease in Pamplona, Norte de Santander, which requires the frequent use of chemical fungicides, although the possibility of using biological control has been informed. This investigation aimed to evaluate the efficacy of biological alternatives for the control of Ascochyta spp. in the cultivation of peas (Pisum sativum) under Pamplona conditions. An experimental investigation was conducted, where six treatments were evaluated: three with bio-preparations produced by the Association of Agricultural Producers (ASPAGRO), alone or in combination, a commercial antagonist (Trichoderma harzianum), a chemical treatment with benomyl, and a control. The incidence of the disease was determined, the Area under the Disease Progress Curve (ABCPE). In addition, the number of pods per plant, the number of grains per pod, the weight of 10 grains and the yield were compared in the treatments. Caldo Rizósfera bio-preparation from ASPAGRO and the commercial Trichoderma-based bio-preparation reduce the incidence and ABCPE of the Ascochyta spp. complex. in peas similar to the chemical fungicide benomil, obtaining crop yield levels similar to those of the chemical treatment.
\end{abstract}

Key words:

Pisum sativum, Trichoderma, efficient microorganisms, enviroment.

\section{INTRODUCCIÓN}

La arveja (Pisum sativum L.) es un alimento muy apetecido dentro de los consumidores ya que tiene mucho aportes nutricionales y beneficios para la salud. (Pabón y Zapata, 2012). Es considerada como uno de los productos básicos de la economía campesina de pequeños y medianos productores, ubicados especialmente en la zona andina. En Colombia la arveja es la leguminosa que ocupa el segundo lugar en orden de importancia después del fríjol, por el área cultivada y por ser fuente de proteína, $(22 \%$ y $25 \%)$, constituyéndose en un alimento básico de la canasta familiar (Cáceres y Gelves, 2013; Angulo et al., 2017).

El cultivo de la leguminosa presenta riesgos de pérdidas en la cosecha debido a las altas precipitaciones propias de la zona andina, que crean condiciones favorables para el desarrollo de patógenos fungosos, principalmente del género Ascochyta, los cuales adquieren importancia económica por el daño que causan a los órganos aéreos de la planta como hojas, tallos y vainas presentando un grave problema en la comercialización en vaina verde, afirmándose por Tamayo (2000) que la arveja es muy sensible a tres especies del género Ascochyta.

En Lituania $A$. pisi era la especie predominante y representaba casi la mitad de los patógenos aislados del complejos Ascochyta, mientras que en algunos sitios, las especies prevalentes eran $A$. pinodes y $P$. pinodella (Cesnuleviciene et al., 2014). De forma similar se ha informado en China que $A$. pinodes y $P$. pinodella son los patógenos mas concurrentes (Liu et al., 2016).

En Colombia se ha encontrado resistencia de líneas de alverja a $A$ pisi y $A$ pinodes, pero ninguna superó el rendimiento de los testigos (Valencia et al, 2012; Meneses et al., 2016). Para el control del complejo Ascochyta se usan generalmente fungicidas a base de Antracol 70 PM, Baycor 300 EC, Cuprosan 311 Super D, Zineb 75\%. (Tamayo, 2000), los cuales contamina el medio ambiente y las vainas y los granos cuando se comercializan verde.

En relación al control biológico se plantea que la aplicación de con Trichoderma harzianum mejora la germinación, la salud y rendimiento de las plantas y también las 
protegió eficazmente las semillas, las plántulas y las plantas adultas de la infección por hongos del suelo entre ellos Ascochyta pisi. (Pięta, y Pastucha, 2009).

En Pamplona que se comercializan bioproductos a base de Trichoderma además se producen bioproductos a bases de microorganismos eficientes caracterizados por su actividad anatagonista contra microoganismos patógenos (Velandia et al., 2016), y que en la Finca Sol Vida también se producen y se comercializan a nivel local bioproductos a partir de microorganismos eficientes con potencial efecto antagonistas (Melo et al., 2017; Castellanos et al., 2018) solo se aplican solo fungicidas químicos para el control del complejo Ascochita. Peñaranda y Molina, (2011) en una investigación desarrollada sobre la arveja en la Vereda Monteadentro, Pamplona Norte de Santander recomendaron el empleo de alternativas naturales para el control de enfermedades con el objetivo de disminuir el excesivo empleo de agroquímicos, que al final contaminan el rio Pamplonita principal colector de las aguas fluviales de esa cuenca (Ortega, 1916).

El objetivo de la presente investigación fue evaluar la eficacia de alternativas biológicas para el control de Ascochyta spp. en el cultivo de arveja (Pisum sativum) en las condiciones de Pamplona, Norte de Santander

\section{METODOLOGIA}

La investigación se desarrolló en Pamplona en el periodo comprendido de marzo a agosto de 2017 La investigación en campo de desarrolló en una parcela de alverja en la Vereda Monteadentro en el Municipio Pamplona, Norte de Santander, y los laboratorios de microbiología y del Centro de Investigación de Sanidad Vegetal e Bioinsumos (CISVEB) de la Universidad de Pamplona donde concurrieron las siguientes condiciones meteorológicas de temperatura, humedad relativa y lluvia:

Se condujo experimento dirigido al control del complejo Ascochyta, teniendo en cuenta que esta es una de las enfermedades con mayor incidencia en alverja en la Vereda Monteadentro del Municipio Pamplona. El montaje del experimento se efectuó en la finca La Cruz, en un lote donde el mes anterior se había observado una fuerte afectación por el complejo Ascochyta en la base del tallo en un cultivo de arveja.

Se evaluaron seis tratamientos: tres con dos biopreparados de ASPAGRO solos 0 combinados, uno biológico con un biopreparado comercial de Trichoderma harzianum TRICHOX WP $5 \times 10^{8}$ ufc/g de la firma BIOX, un tratamiento químico con benomil (Benomyl 50 WP) de la firma Del Monte y un testigo sin tratamiento.:

Los biopreparado fueron Microorganismos de Montaña (MM) y Caldo Rizósfera (CR). Los ingredientes de los mismos, y recomendación de uso aparecen en la siguiente Tabla 2.

Tabla 1. Concentracion de los biopreparados

\begin{tabular}{|l|l|l|}
\hline Biopreparados & Ingredientes & Uso propuesto \\
\hline MM & $\begin{array}{l}\text { ME, melaza y } \\
\text { salvado de arroz. }\end{array}$ & $\begin{array}{l}\text { Biofertilizante } \\
\text { con B.S.F. y } \\
\text { antagonista } \\
1,72 \times 10^{6} \\
\text { UFC/Ml }\end{array}$ \\
\hline $\begin{array}{l}\text { Caldo } \\
\text { Rizósfera }\end{array}$ & $\begin{array}{l}\text { Raíces de plantas } \\
\text { conseguidas en la } \\
\text { granja, yogurt, } \\
\text { melaza, agua } \\
\text { oxigenada y harina } \\
\text { de frijol. }\end{array}$ & $\begin{array}{l}\text { Biofertilizante } \\
\text { con B.S.F. y } \\
\text { antagonista } \\
1,24 \times 10^{6} \\
\text { UFC/Ml }\end{array}$ \\
\hline
\end{tabular}

Los tratamientos se realizaron cada 14 días dirigidos al suelo y a la parte inferior de las plantas en horas de la tarde. Estos se hicieron con una bomba manual de espalda de $20 \mathrm{~L}$ litros de capacidad. Las dosis de aplicación de cada tratamiento se relacionan en la Tabla 3, siempre aplicados a 400L/ha. 


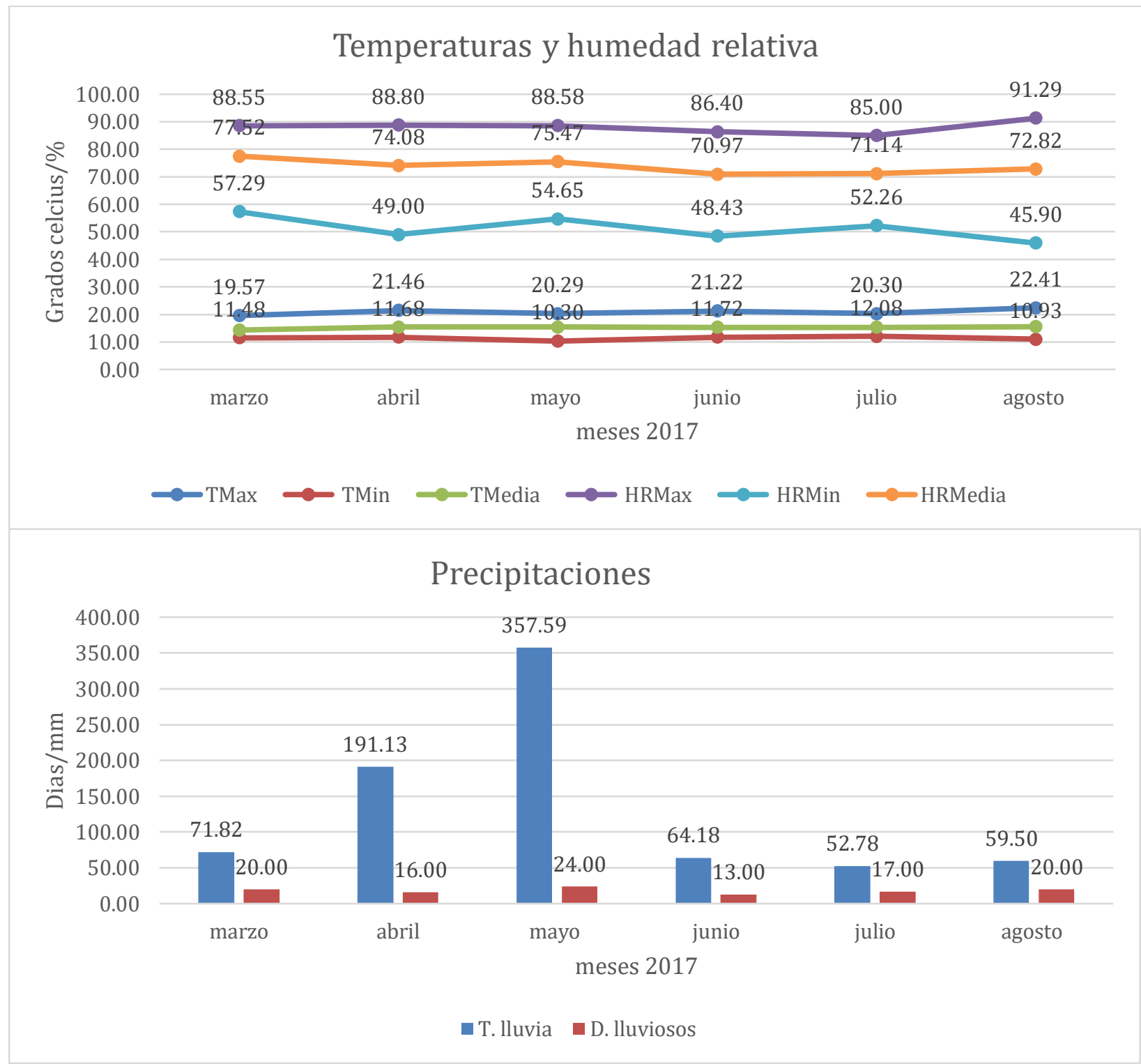

Figura 1. Condiciones meteorológicas concurrentes durante el desarrollo del cultivo Fuente: Elaboración propia a partir de la información de la Estación del CISVEB

Tabla 3. Dosis de aplicación y solución final

\begin{tabular}{|c|c|}
\hline Producto & Dosis \\
\hline $\begin{array}{l}\text { M. de Montaña } \\
\text { (MM) }\end{array}$ & $1 \mathrm{~L} / 20 \mathrm{~L}$ \\
\hline Caldo Rizósfera & $1 \mathrm{~L} / 20 \mathrm{~L}$ \\
\hline $\begin{array}{l}\text { MM + Caldo } \\
\text { Rizósfera }\end{array}$ & $1 \mathrm{~L} / 20 \mathrm{~L}+1 \mathrm{~L} / 20 \mathrm{~L}$ \\
\hline Trichoderma & $0,8 \mathrm{~g} / 20 \mathrm{~L}$ \\
\hline Fungicida benomil & $\begin{array}{l}0,5 \mathrm{~g} / 20 \mathrm{~L}(250-500 \\
\mathrm{g} / \mathrm{ha})\end{array}$ \\
\hline
\end{tabular}

Los tratamientos se realizaron cada 14 días dirigidos al suelo y a la parte inferior de las plantas en horas de la tarde. Estos realizaron con una bomba manual de espalda de $20 \mathrm{~L}$ litros de capacidad. Se realizaron los tratamientos con una asperjadora manual de espalda

Se realizaron labores de preparación del suelo y se programó una fertilización antes de la siembra incorporando $1 \mathrm{~kg}$ de urea, 1 $\mathrm{kg}$ de cloruro de potasio y $5 \mathrm{~kg}$ de 10-30- 
10; se empleó la variedad "Rabo Gallo" de gran arraigo en la zona, sembrada directamente en el campo en surcos separados entre sí a una distancia de 120 $\mathrm{cm}$ y $2 \mathrm{~cm}$ entre surcos y plantas, respectivamente. El sistema de tutorado se estableció 20 días después de la siembra (dds) y un segundo tutor se colocó cuando las plantas tenían una altura promedio de $30 \mathrm{~cm}$.

Se empleó un diseño bloques al azar con cuatro réplicas $6 \times 4$ (6 tratamientos con 4 réplicas), 0 sea, 24 unidades experimentales (parcelas). El tamaño de cada parcela experimental fue de 5 metros de largo por 4 metros de ancho con un área de $20 \mathrm{~m}^{2}$. La distancia entre plantas fue de $2 \mathrm{~cm}$ y entre surcos de $1 \mathrm{~m}$.

Para determinación de la incidencia del complejo Ascochyta y el comportamiento de los tratamientos en el control de la enfermedad, se tomaron 20 plantas al azar por cada una de las parcelas, en los surcos centrados tres días después de cada aplicación evaluándose las plantas enfermas por Ascochyta sobre el total evaluadas, cada 15 días. Con esta información se calculó la incidencia por la formula siguiente:

$\%$ de incidencia $=\frac{n(\text { plantas afectadas })}{N \text { (evaluadas })} X 100$

(Ecuación 1) (Agrios, 2006)

Se tomaron muestras de las plantas enfermas y se llevaron ala laboratorio de microbiología para confirmar el agente presente en los principales síntomas que se observaban.

Al final del experimento se determinó el Área Bajo la Curva de Progreso de la Enfermedad (ABCPE), para lo cual se utilizó la siguiente formula:

ABCPE $=\Sigma\left[\left(\mathrm{X}_{\mathrm{i}}+\mathrm{X}_{\mathrm{i}}+1\right) / 2\right] *\left(\mathrm{~T}_{\mathrm{i}}+1-\mathrm{Ti}\right)$ (Ecuación 2) (Campbell y Madden, 1990) Donde:
$X_{i=}$ distribución 1 o intensidad 1 de la enfermedad en el muestreo $i$

$X_{i+1}=$ distribución 0 intensidad de la enfermedad en el muestreo $i+1$

$T i=$ tiempo 1

$T_{i+1}=$ tiempo 2

Al final del experimento se evaluaron los indicadores de rendimiento el rendimiento de cada parcela. Los indicadores del rendimiento evaluados vainas por planta en la cosecha (en 20 plantas por parcela), granos por vaina (100 vainas por parcela) y peso de las vainas. Para esta última variable se pesaron 100 vainas por parcela para sacar el promedio. Se estimó el peso de la producción por parcela por parcela a partir del peso promedio de las vainas el número de vaina por parcela y el número de plantas por parcela. A partir de la producción y el área se estimó el rendimiento. Se compararon los rendimientos contra el testigo.

Se realizó un análisis de varianza a todas las variables (excepto las productivas) usando el paquete estadístico SPSSS. Se compararon las medias por la prueba de Tukey, con un $5 \%$ probabilidad de error

\section{ANALISIS $Y$ DISCUSIÓN DE RESULTADOS}

Los síntomas del complejo por Ascochyta se detectadas en las hojas bajeras de las plantas del experimento en el quinto muestreo (Figura 2A), y se identificó el agente causal a partir de las estructuras típicas del hongo (Figura 2B y Figura 2C). A partir de ese momento la incidencia se mantuvo en incremento creciente.

La curva de la dinámica del testigo estuvo siempre por encima del resto de los tratamientos. Se destacaron los tratamientos con Trichoderma y el control químico como los de curvas con valores relativos más bajos y dentro de los biopreparados el que presentó la curva con 
valores relativos más bajos fue el Caldo Rizósfera (Figura 3).

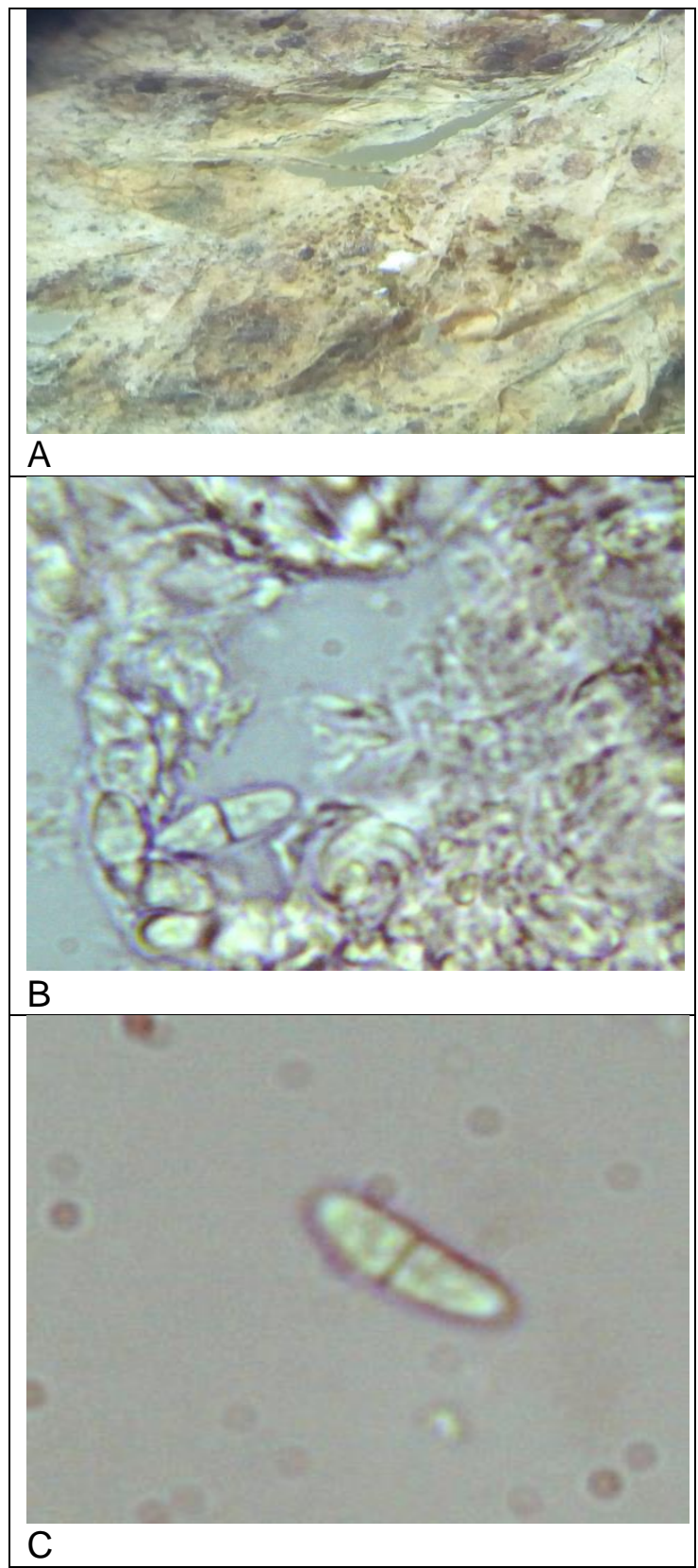

Figura 2. Hoja con síntomas de Ascochyta spp. (A), picnidio del hongo (B) y conidio (C)

El análisis dela ANOVA mostró diferencia estadística entre los tratamientos para el muestreo realizado el 17/04 el valor de incidencia mayor para el testigo $(23,13 \%)$, con diferencia estadística con MM, CR, quedando Trichoderma y $\mathrm{MM}+\mathrm{CR}$ intermedios desde el punto de vista estadístico, sin embargo en el muestreo del 01/05 aunque el testigo tenía el mayor nivel de incidencia, los tratamientos CR, Trichoderma y $\mathrm{MM}+\mathrm{CR}$ de este se diferenciaban de este y no MM (Tabla 3).

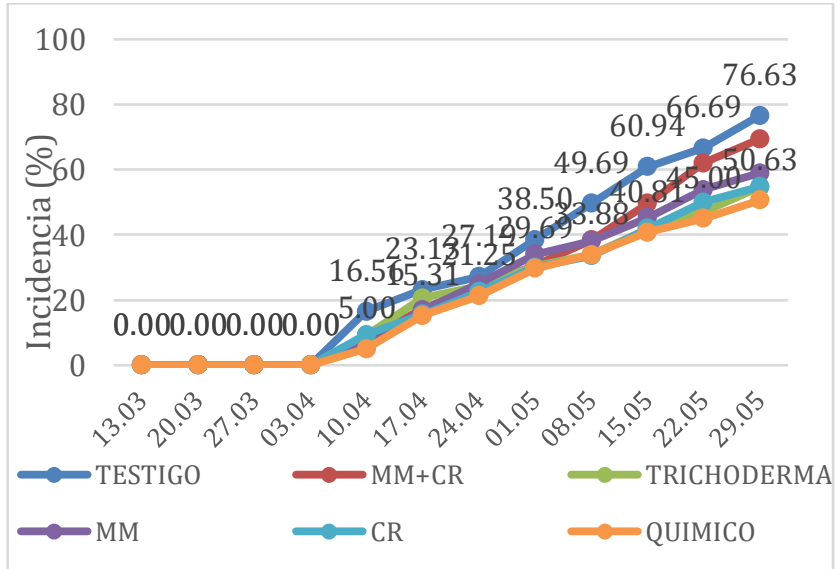

Figura 3. Dinámica de la incidencia del Complejo Ascochyta spp.en el experimento para el control de esta enfermedad en el cultivo de arveja.

Tabla 3. Incidencia y ABCPE del complejo Ascochyta spp. en los tratamientos del experimento para el control de esta enfermedad en el cultivo de arveja

\begin{tabular}{lcccl}
\hline Tratamientos & \multicolumn{4}{l}{ Incidencia (\%) / Fechas } \\
& $\mathbf{1 7 - 0 4}$ & $\mathbf{0 1 - 0 5}$ & $\mathbf{1 5 - 0 5}$ & $\mathbf{2 9 . 0 5}$ \\
\hline Testigo & $23,1 \mathrm{a}$ & $38,5 \mathrm{a}$ & $60,9 \mathrm{a}$ & $76,6 \mathrm{a}$ \\
MM + & $17,38 \mathrm{ab}$ & $30,6 \mathrm{~b}$ & $\mathbf{4 9 , 7} \mathrm{b}$ & $69,4 \mathrm{a}$ \\
C.R. & & & & \\
Trichoderma & $20,6 \mathrm{ab}$ & $30,3 \mathrm{~b}$ & $41,7 \mathrm{c}$ & $54,8 \mathrm{~b}$ \\
MM & $16,8 \mathrm{~b}$ & $34,1 \mathrm{ab}$ & $45,3 \mathrm{bc}$ & $59,0 \mathrm{~b}$ \\
C.R. & $15,6 \mathrm{~b}$ & $30,1 \mathrm{~b}$ & $41,9 \mathrm{c}$ & $54,8 \mathrm{~b}$ \\
Químico & $15,3 \mathrm{~b}$ & $29,7 \mathrm{~b}$ & $40,8 \mathrm{c}$ & $50,3 \mathrm{~b}$ \\
\hline C.V. (\%) & 4,7 & 3,9 & 3,8 & 5,2 \\
Error Típico* & 0,03 & 0,03 & 0,03 & 0,05 \\
\hline
\end{tabular}

* Medias con letras desiguales en las columnas difieren por la prueba de Tukey $(P \leq 0,05)$.

En el muestreo del 15/05 el testigo mostró el mayor nivel de incidencia, todos los tratamientos se diferenciaban de este, sin embargo el control químico, trichoderma, CR y MM no se diferenciaban entre sí, pero sí de $\mathrm{MM}+\mathrm{CR}$, con excepción de MM. En el último muestreo del 29/05, los tratamientos de mayor incidencia del complejo Ascochyta en la base del tallo fueron el testigo con $76,62 \%$ y $\mathrm{MM}+\mathrm{CR}$ con diferencia estadística con el resto de los tratamientos. 
Un estudio en 2017 confirmó que Trichoderma spp. inhibe el crecimiento radial de Ascochyta pinodella (76.9\%), A. pinodes $(60.0 \%)$ y $A$. pisi $(68.5 \%)$ y a pesar de los resultados anteriores estos autores declararon que los resultados eran los primeros informes de la eficacia in vitro de Trichoderma spp. contra A. pisi, A. pinodes, A. pinodella (Živanov et al., 2017).

El ABCPE resultó mayor desde el punto de vista estadístico para el testigo, todos los tratamientos se diferenciaban de este, sin embargo, el control químico, Trichoderma y CR no se diferenciaban entre sí. El ABCPE MM y MM + CR no se diferenció desde el punto de vista estadístico, pero MM se diferenció del químico y no de MM y CR (Tabla 4).

Tabla 4. .ABCPE de la incidencia del complejo Ascochyta spp. en los tratamientos del experimento para el control de esta enfermedad en el cultivo de arveja

\begin{tabular}{ll}
\hline Tratamientos & ABCPE \\
\hline Testigo & $4494,00 \mathrm{a}$ \\
MM + Caldo rizósfera & $3705,18 \mathrm{~b}$ \\
Trichoderma & $3282,56 \mathrm{~cd}$ \\
MM & $3503,50 \mathrm{bc}$ \\
Caldo rizósfera & $3227,43 \mathrm{~cd}$ \\
Químico & $3027,50 \mathrm{~d}$ \\
\hline C.V. (\%) & 5,0 \\
Error Típico* & 90,24 \\
\hline
\end{tabular}

* Medias con letras desiguales en las columnas difieren por la prueba de Tukey $(P \leq 0,05)$.

Los controles que se llevan a cabo en los cultivos de hoy en día se están volviendo algo de gran importancia puesto a que se buscan medidas de control que ayuden a mitigar las enfermedades primarias de los cultivo, las casas comerciales ofrecen productos químicos que ayudan a contrarrestar esas enfermedades pero en realidad lo que hacen es posponer el desarrollo de estos patógenos y que más adelante vuelvan y ataquen con mayor rigor puesto a que se vuelven resistente a estos productos comerciales. Entonces las nuevas alternativas que se buscan es el control biológico pero los productores no toman esta alternativa porque creen que son insuficiente para el control de enfermedades, pero se sabe que los productos biológicos no solo actúan para el control de enfermedades, además que aportan beneficios nutricionales a plantas y suelo que se verán reflejados a lo largo del tiempo. Liu et al., (2016) informan que entre más de 100 aislados de bacterias tres cepas presumiblemente correspondientes a Pantoea agglomerans,. Bacillus amyloliquefaciens $y$ Bacilus. Subtilis demostraron tener un efecto antagonico fuerte contra $A$. pinodes y $P$. pinodella

Con relación a los componentes del rendimiento los granos/vaina variaron entre 8 y 9 para los tratamientos biológicos solos y el químico, pero disminuyó a 7 y 6,2 para la mezcla $\mathrm{MM}+\mathrm{CR}$ y el testigo respectivamente, mientras que las vainas/plantas variaron entre 17 y 18 para el tratamiento químico y los biológicos solos, y bajo a 13 para $\mathrm{MM}+\mathrm{CR}$ y a 10,45 en el testigo y de forma similar se comportó la variable peso de las vainas g/vaina con valores muy similares y relativamente más altos para para los tratamientos biológicos solos y el químico, intermedio para MM + CR y menor para el testigo (Figura 4).

Los rendimientos solo alcanzaron 3,75 t/ha en el testigo y 5,2 $\mathrm{t} / \mathrm{ha}$ en el tratamiento MM $+\mathrm{CR}$, mientras que en el resto oscilaron entre 7,43 y 7,76 t/ha. Los mayores rendimientos relativos se obtuvieron en el control químico y Trichoderma (7,76 t/ha), aunque MM y CR alcanzaron más del $94 \%$ de este valor, sin embargo, el tratamiento de $\mathrm{MM}+\mathrm{CR}$ solo alcanzó el $66,3 \%$ de ese valor y el testigo 46,44\% (Figura 5).

Una respuesta positiva es la utilización de microorganismos antagónicos competitivos para proteger los cultivos de patógenos del suelo; en particular especies del género Trichoderma han merecido la atención 
máxima como agente de biocontrol (Rosero, 2008).

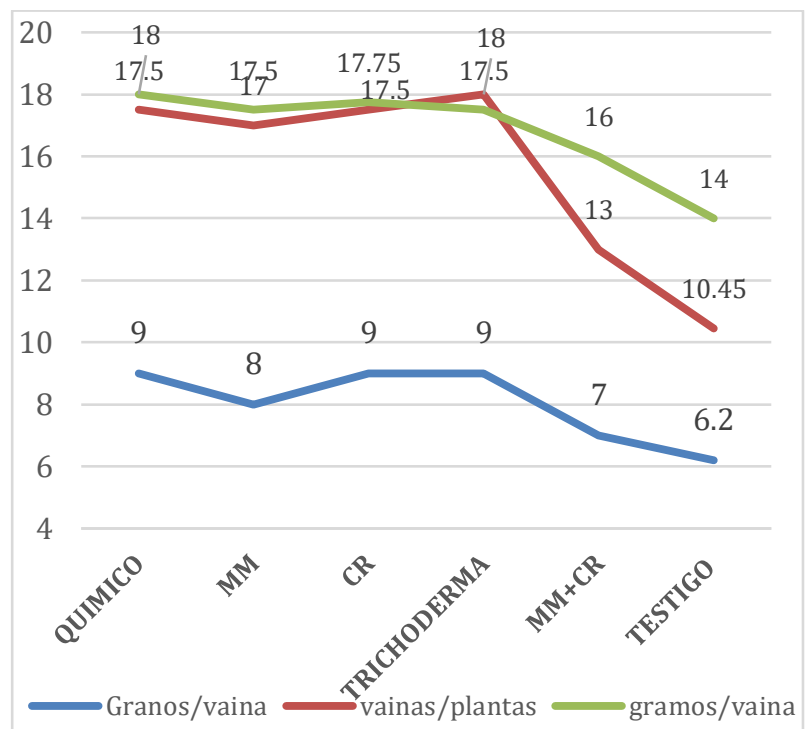

Figura 4. Componentes del rendimiento en los diferentes tratamientos en el experimento de biopreparados contra del complejo Ascochyta spp.

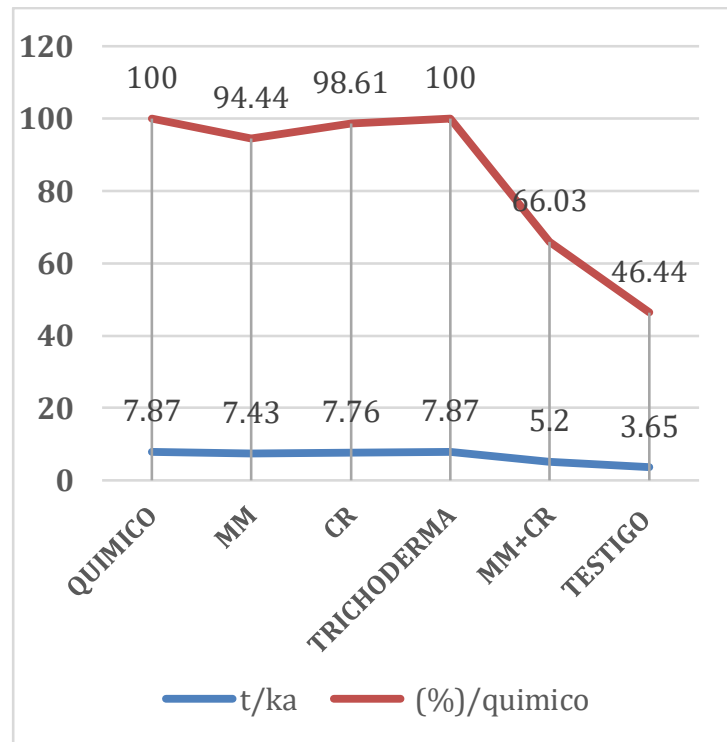

Figura 5. Rendimiento en los diferentes tratamientos en el experimento de biopreparado contra del complejo Ascochyta spp. y porcentaje que representa respecto al químico.

Estos resultados en los rendimientos se corroboran con los niveles de control obtenidos anteriormente para el complejo
Ascochyta por el fungicida químico, el biopreparado comercial Trichoderma y los dos biopreparado de ASPAGRO aplicados por separado (MM y CR).

Se pone de manifiesto el efecto que hace la enfermedad sobre los componentes del rendimiento cuando no se hace un control eficaz. Por otra parte, en todos los tratamientos estos indicadores presentaron valores relativos inferiores a los mostrados en el experimento para el control del complejo Ascochyta.

Ya otros autores han señalado que los tratamientos a las semillas de alverja con conidios de Trichoderma harzianum sus líquidos post-cultivo mejoran las emergencias, la salud y rendimiento de las plantas y también las protegió eficazmente de la infección por hongos del suelo entre ellos Ascochyta pisi. (Pięta, y Pastucha, 2009).

Por otra parte, ya se conocía que la aplicación de Trichoderma sp. comercial en el cultivo de arveja mejora notablemente su crecimiento y desarrollo, influyendo en variables fisiológicas como germinación, área foliar, peso seco y fresco de la raíz, peso seco y fresco de la parte aérea, y longitud de raíz (Camargo-Cepeda y Ávila, 2014).

En ensayos de control usando Trichoderma para los tratamientos biológicos en la variedad de arveja Santa Isabel se obtuvieron 6,50 y 7,45 granos por vaina (Eraso et al. 2014; Camargo, 2016), lo cual se corresponde con los resultados del presente trabajo.

\section{Conclusiones}

Se comprueba que el biopreparado Caldo Rizósfera de ASPAGRO y el biopreparado comercial a base de Trichoderma logran reducir la incidencia y el ABCPE del complejo Ascochyta spp. en arveja de 
forma similar al fungicida químico benomil, obteniéndose niveles de rendimiento del cultivo similares a los del tratamiento químico.

\section{Referencias Bibliográficas}

Agrios G. 2006. Plant disease. New York. American Phytopathology Society. Vol. 98, no 6, p. 1117-1128.

Angulo, W. J., Mendoza, J. A. y Uriel, H. U. (2017). Análisis de la vulnerabilidad por fenómenos de remoción en masa en la Cuenca Tanauca estudio de caso. Revista Ambiental Agua, Aire y Suelo. ISSN 19009178. Volumen (8), Numero (2). DOI: https://doi.org/10.24054/19009178.v2 .n2.2017.3276

Cáceres, G. P., \& Gelves, D. Y. M. (2013). La producción de arveja Pisum sativum en la vereda Monteadentro, provincia de Pamplona, Norte de Santander. Face: Revista de la Facultad de Ciencias Económicas y Empresariales, 11(2).

Camargo-Cepeda D.F. y Ávila, E.R. 2014. Efectos del Trichoderma sobre el crecimiento y desarrollo de la arveja (Pisum sativum). Ciencia y Agricultura 1 (1), 91100.

Camargo, W. C. (2016). Modelación hidrologico-hidraulica de eventos de inundacion en el Rio Bogotá (sector tocanzipa-chia) usando HEC-RAS. Revista Ambiental Agua, Aire y Suelo. ISSN 19009178. Volumen (7), Numero (2). DOI: https://doi.org/10.24054/19009178.v2 . n2.2016.3267

Campbell CL. and Madden LV. (1990). Introduction to Plant Disease Epidemiology. New York: John Wiley \& Sons, $532 \mathrm{p}$.

Castellanos, L., Céspedes, N., Sequeda, A., Jaime, J.E., y Niño, L.J. 2018. Caracterización microbiológica de cinco biopreparados artesanales. Revista Científica Agroecosistemas. 6(3): 57-65.
Cesnuleviciene, R., Gaurilcikiene, I., and Ramanauskiene, J. 2014. Control of ascochyta blight (Ascochyta complex) in pea under Lithuanian conditions. Zemdirbyste 101, 101-108. doi: 10.13080/z-a.2014.101.01

Eraso, C., Acosta, J., Salazar, C., Betancourth, C. 2014. Evaluación de cepas de Trichoderma spp. para el manejo del amarillamiento de arveja causado por Fusarium oxysporum. Corpoica. Ciencia y Tecnología Agropecuaria.15 (2): 237-249.

Liu, N. ,Xu, S., Yao, X., Zhang, G., Mao, W., Hu, Q., Feng Z. and Gong Y. 2016. Studies on the Control of Ascochyta Blight in Field Peas (Pisum sativum L.) Caused by Ascochyta pinodes in Zhejiang Province, China. Frontiers in microbiology. $7 . \quad$ Art. 481. https://doi.org/10.3389/fmicb.2016.00481

Melo, J., Saavedra, S. y Ramón, J. A. (2017). Evaluación de la adsorcion de $\mathrm{CU}+2$ y azul de metileno en biosorbentes de bajo costo obtenidos a partir de biomasa residual de la agroindustria de cítricos. Revista Ambiental Agua, Aire y Suelo. ISSN 1900-9178. Volumen (8), Numero (2). DOI: https://doi.org/10.24054/19009178.v2 .n2.2017.3277

Meneses, V. B., Álzate, D. y Mosquera, J. (2016). Sistema de optimización de las técnicas de planificación en agricultura de precisión por medio de drones. Revista Ambiental Agua, Aire y Suelo. ISSN 19009178. Volumen (7), Numero (2). DOI: https://doi.org/10.24054/19009178.v2 .n2.2016.3268

Ortega, J. Y. 2016. Estructura ecológica principal de la cuenca del Río Pamplonita. Revista Ambiental Agua, Aire y Suelo. ISSN 1900-9178. Volumen (7), Numero (1). DOI: https://doi.org/10.24054/19009178.v1 .n1.2016.3258

Peñaranda, G., Molina, D.Y. 2011. La producción de arveja (Pisum sativum) en la vereda Monteadentro, provincia de 
Pamplona, Norte de Santander. Revista FACE. 8(11):43-56.

Pięta, D., Pastucha A.. 2009. The effectiveness of antagonistic fungi and their post-culture liquids in pea (Pisum sativum $\mathrm{L}$.) protection against diseases. Acta Sci. Pol., Hortorum Cultus 8(1) 2009, 13-25

Tamayo, P.J. 2000. Enfermedades del cultivo de la arveja en Colombia: Guía de reconocimiento y control. Ministerio de Agricultura y Desarrollo Rural, Corpoica, Fenalce, Sena y SAC. p. 49.

Valencia, A., Timaná, Y., \& Checa, O. (2012). Evaluacion de 20 lineas de arveja (Pisum sativum $L$ ) y su reacción al complejo de Ascochyta. Revista de Ciencias Agrícolas, 29(2), 39-52.

Velandia, F. J., Granados, J. D., Ramón, J. D. y Roa, A. L. 2016. Caracterización de consorcios microbianos con potencial degradador de contaminantes en el municipio de Pamplona, Norte de Santander. Revista Ambiental Agua, Aire y Suelo. ISSN 1900-9178. Volumen (7), Numero

DOI: https://doi.org/10.24054/19009178.v1 . $\mathrm{n} 1.2016 .3278$

Živanov S T., Radivoje Jevtic R.,, Mirjana Lalošević, M., Živanov, D. Medic-Pap, S., Zupunski, V. 2017. Efficacy of Trichoderma spp. against Common Fungal Pathogens. Ratar. Porvt. 54 (3), 104, 109. DOI: $10.5937 /$ ratpov54-14254 